\title{
EFFECT OF NONCONFORMANCES ON THE AERODYNAMIC PERFORMANCE OF LOW-PRESSURE TURBINE OUTLET GUIDE
}

\section{Chernoray ${ }^{1}$, R. Ramos Alonso ${ }^{1}$, J. Larsson $^{2}$, and S. Ore ${ }^{2}$}

${ }^{1}$ Department of Applied Mechanics Chalmers University of Technology

41296 Göteborg, Sweden

${ }^{2}$ Department of Aero and Thermo Dynamics

Volvo Aero Corporation 46181 Trollhättan, Sweden

Geometry nonconformances are diverse variations in the actual component geometry with respect to the intended ideal design. These differences in the geometry can occur either at component manufacturing or later during the exploitation as a result of component wear and reparations. Typical geometry nonconformances are nonideal shapes and positions due to tolerances, manufacturing traces such as shrinkage, warps, welds, repairs of various type, e.g., patches, and other shape deviations such as surface roughness. There are also so-called nominal nonconformances which include, e.g., steps, gaps, fillets, bumps, and installation holes. Improvement of the aerodynamic component performance requires understanding of the impact of these technological features on the fundamental flow field. In the current study, experiments and computational fluid dynamics (CFD) simulations are performed on two different types of nonconformances on the outlet guide vanes in order to study the effect of the nonconformances on the aerodynamic performance and to validate the computation design tool.

\section{INTRODUCTION}

Geometry nonconformance effects have long been neglected and handled as a second-order problem. Engine designers assumed a perfect geometry and often put unrealistic requirements to the final geometry in order to ensure that any real geometry effects will not interfere with their ideal design. Due to demands on enhanced performance, reduction of manufacturing costs, and increased engine life with longer service intervals, the study of the geometry nonconformance

This is an Open Access article distributed under the terms of the Creative Commons Attribution-Noncommercial License 3.0, which permits unrestricted use, distribution, and reproduction in any noncommercial medium, provided the original work is properly cited. 
effects have become important. For example, there is a tendency for core compressors to decrease their size by increasing the engine by-pass ratio. However, the smaller compressors are expected to have similar size of manufacturing deviations, damages, and tolerances. This means that the real geometry problems which previously were negligible are now becoming significant and create new type of problems for engineers. Another important example is that many new advanced technologies used in aerodynamic design, such as nonaxisymmetric end-walls, highly loaded blades and vanes, and superaggressive intermediate ducts are usually performing very well in theory and work good in experiments, but often run into problems due to real-geometry defects that are present in real engines or as the engine deteriorates.

Since the geometry nonconformances have high impact on engine-component cost and durability, the aerodynamic design engineers have come to a conclusion that designing an ideal geometry is no longer sufficient. Nonideal effects need to be taken into account early in the design process in order to obtain a robust design with good performance and acceptable variability between individual components and different engines. This means that computational design and analysis methods are needed to account for these problems during the design phase, and these computational tools must be developed and validated.

Volvo Aero's civil "design-make" specialization is the static structures of airbreathing engines. There are several structural frames in the engine, so-called TEC/TRF/TBH (rear frame structure), TCF/MTF (central frame of turbine), and IMC/FHF/ICC (compressor frame). The subject of this study is the rear frame structure. The TRF components are referred to as low-pressure turbine outlet guide vanes (LPT/OGVs). The OGVs are located in the last part of the engine internal flow path just before the nozzle. The main aerodynamic function of the $\mathrm{OGVs}$ is to deswirl the flow coming from the last low-pressure turbine rotor into an axial outflow. The OGVs also contain support services such as electrical control lines and oil lines connected to the rear low-pressure shaft bearing. Both the high structural loads and the relatively large support lines passing through them make the OGVs thick and hence challenging to optimize in terms of aerodynamic losses and flow separation. The problem of surface nonconformance is of high importance for OGVs since these large structural components are usually not replaced and subjected to long-time erosion, potential damages and repairs during the whole engine lifetime. It appears that some production and in-service defects of the OGVs can influence the aerodynamic performance considerably while other can be considered as unimportant and tolerated. The latter means a reduction in the production cost.

Presented in this study are the experiments and CFD calculations with a highly loaded LPT/OGV cascade equipped with simulated surface geometry variations. Two major types of the OGV surface nonconformances were simulated and investigated: a two-dimensional (2D) welding trace and a three-dimensional (3D) bump (bulge). The experimental studies were performed in a large-scale 
linear cascade facility at Chalmers University. Computations by two different turbulence models, $k-\omega$ shear stress transport (SST) and realizable $k-\varepsilon$ were carried out and validated. Load distributions, losses, outlet flow angles, and the evolution of the secondary flow-field were extensively monitored and analyzed.

\section{EXPERIMENTAL SETUP}

Experiments were performed in a linear cascade facility at Chalmers University. This facility is of open circuit blower type and operates in the range of realistic Reynolds number for OGV flows. Design and validation of the facility was described in detail by Hjärne et al. [1, 2] and Hjärne [3]. The incoming flow turbulence intensity was varied by a turbulence grid placed upstream of the cascade (Fig. 1). Two traversing systems have been used to measure the flow both upstream and downstream. Typical measurement planes are shown in Fig. 1. The traversing systems were controlled by stepper motors and all measurements were performed in an automated mode.

Measurements of the three flow velocity components and pressure were performed by multihole pressure probes. A five-hole probe was mounted in the upstream location. The diameter of the five-hole probe head was $3.5 \mathrm{~mm}$ with individual distances between the holes of $1 \mathrm{~mm}$ and the tip half-cone angle of $45^{\circ}$. A seven-hole probe was used in the downstream location. The diameter of the seven-hole probe head was $2 \mathrm{~mm}$ with individual distances between the holes of $0.5 \mathrm{~mm}$ and the tip half-cone angle of $30^{\circ}$. During the measurements, the pres-

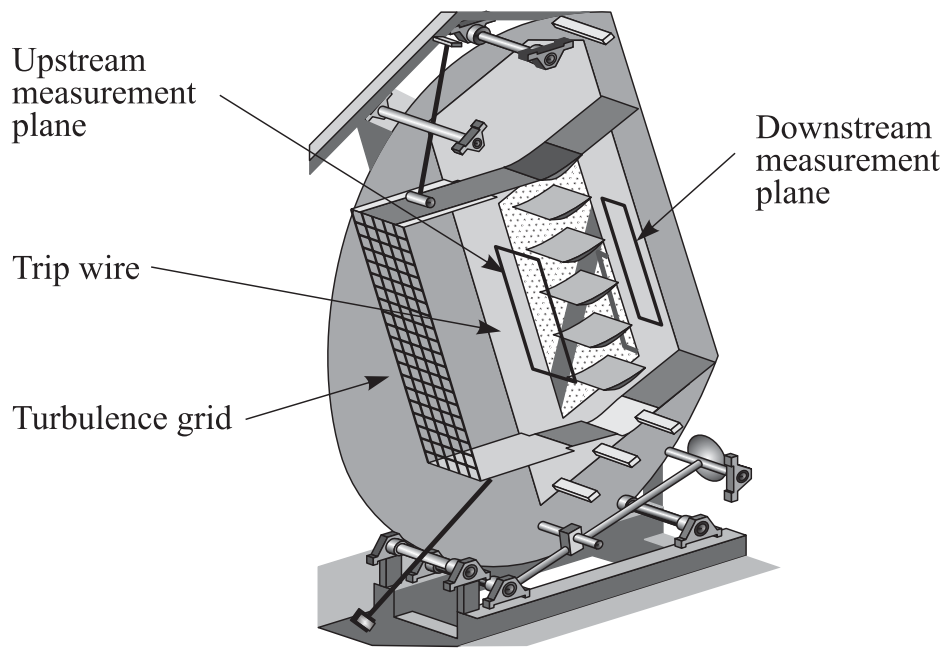

Figure 1 Linear LPT/OGV cascade facility 
sures were monitored by a 16-channel PSI 9116 digital pressure scanner (Pressure System Inc.). At each spatial point, 1000 data samples were taken and the mean pressures were recorded. The sampling settings resulted in a statistical uncertainty of $0.8 \%$ with 99 percent confidence for regions with the highest fluctuations of pressure (10\%). The procedure of probe calibration and applied correction for the velocity gradient effects was described in [4]. Postprocessing of experimental data was performed using Matlab software.

\section{NONCONFORMANCES}

Investigated nonconformances included specially designed $2 \mathrm{D}$ and $3 \mathrm{D}$ surface modifications which simulated a 2D generic welding trace and a $3 \mathrm{D}$ surface repair patch. The relative size of the surface modification elements was chosen fairly large, of order of $10 \%-20 \%$ of the blade maximum thickness. Figure 2 shows the

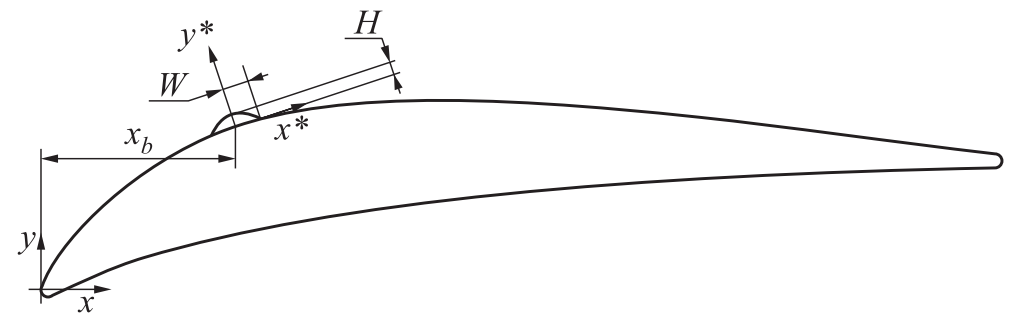

Figure 2 Geometry definition of simulated 2D welding trace

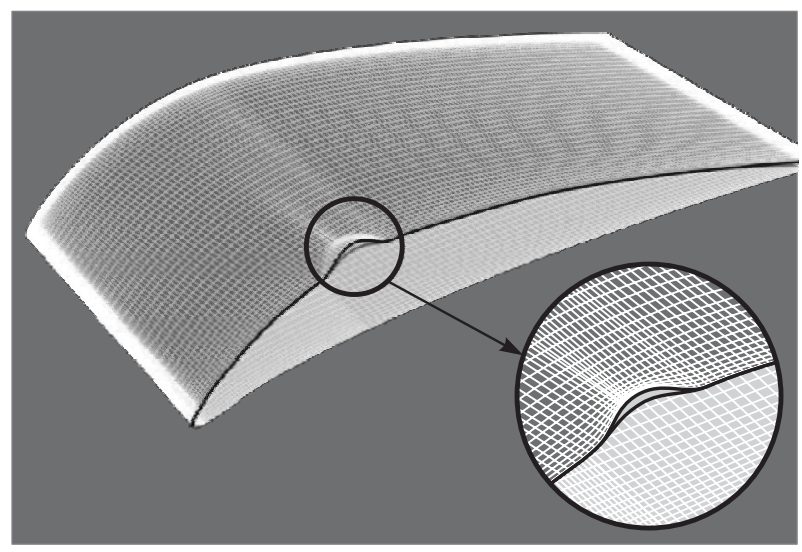

Figure 3 Surface mesh and geometry of 3D bump 
parameters which define the $2 \mathrm{D}$ welding trace. The height of the weld, $H$, is $2.5 \mathrm{~mm}$, and the half-width, $W$, is $6 \mathrm{~mm}$. Further in text, the weld position on the vane, $x_{b}$, is expressed normalized by the vane chord length as $x / c$. Numerical simulations were performed for the cases of weld positions at $x / c=0.05,0.1$, $0.2,0.3$, and 0.4 .

Figure 3 shows the shape of a 3D bump. The bump is a body of revolution with a cross section defined as a fourth order curve. This bump was placed on the surface of the vane so that it was bending along the surface. In experiments, this was achieved by using a flexible material for the bump. Bumps of two different dimensions (height $\times$ diameter) were used: $5 \times 20$ and $3 \times 20 \mathrm{~mm}$.

\section{NUMERICAL SETUP}

The goal of numerical studies was to choose simulation methods for an accurate and reliable prediction of the LPT/OGV performance with geometry variations. The flow in the OGV cascade was highly diffusive with a presence of strong secondary flows and growing boundary layers. These effects and risk of separation were increased with introducing the surface nonconformances, and the calculations with turbulence models became essentially challenging. From previous works [1-3], the $k-\varepsilon$ realizable and $k-\omega$ SST turbulence models were known to perform well for the OGV cascade and, therefore, these models were selected for the current study. Main parameters of the numerical simulations and the boundary conditions at the inlet, such as velocity, turbulence length scale, turbulence intensity, and sidewall boundary layer thickness, were matched with the experimental conditions in the linear cascade. The calculations were steady and incompressible. The reference cases without nonconformances in $2 \mathrm{D}$ and $3 \mathrm{D}$ were implemented first. The calculations of the simulated welding traces were performed in 2D and the calculations of the bulges were in 3D. For all cases, different meshes were implemented to study mesh dependency. ANSYS ICEM v. 11 was used for meshing. Structured grids were created and the vane was surrounded by an O-grid with resolved boundary layers. For 2D cases, the mesh size was $(50-90) \cdot 10^{3}$ cells, and for $3 \mathrm{D}$ cases, $1.5-2.5$ million cells. Fluent 6.3 was used as a CFD solver.

\section{TURBULENCE LENGTH SCALE STUDY}

The turbulence parameters at the inlet for numerical simulations ought to match the corresponding characteristics in the rig. Of particular difficulty was to obtain experimentally a condition for turbulence dissipation. The preliminary investigations showed an importance of having this value defined correctly since noticeable 


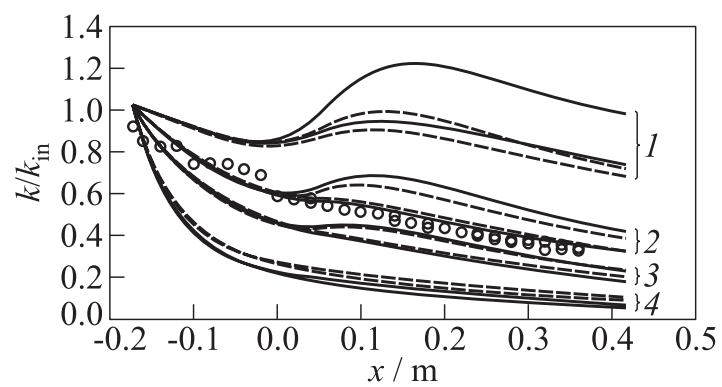

Figure 4 Turbulent kinetic energy in the cascade free stream from the inlet to the outlet. Symbols show the results from hot-wire measurements. Lines show the results of computations with different length scales: $1-5 \mathrm{~mm} ; 2-2 ; 3-1.2$; and $4-$ $0.5 \mathrm{~mm}$. Solid lines used for $k-\varepsilon$ realizable data and dashed lines for $k-\omega$ SST data

discrepancies in the flow behavior and pressure loss coefficient for different turbulence models and different values of the turbulence dissipation were observed. Moreover, at certain values of the inlet dissipation, the $k-\varepsilon$ turbulence model was observed to produce an excessively growing turbulent kinetic energy in the free stream.

Many different methods were investigated for obtaining a correct value of turbulence dissipation in the facility. Among them are such as described in [58]. However, none of these methods was accepted as sufficiently robust and reliable for current purpose. As an alternative, the turbulence dissipation was elaborated from the streamwise development of the turbulent kinetic energy, $k$, in the free stream regions of the flow. The $k$ values were measured by a cross hotwire probe along two parallel lines in the free stream, which were extending from the inlet, between the vanes, and all the way to the outlet. Also, several CFD simulations were performed for different length scales. The kinetic energy decay was compared with the experimental data to obtain the best overall distribution of $k$ (Fig. 4) and in this way, the length scale was defined as $1.2 \mathrm{~mm}$. This method was found sufficiently robust and reliable for obtaining the turbulence dissipation boundary condition.

\section{RESULTS AND DISCUSSION}

Results for the reference "clean" case are not presented here. However, it can be noted that the calculations revealed good flow field predictions with both $k-\varepsilon$ realizable and $k-\omega$ SST turbulence models. The analysis of the total pressure loss coefficient, flow turning, and pressure distribution on the vane revealed good agreement with the corresponding experimental data (see details in [9]) and 


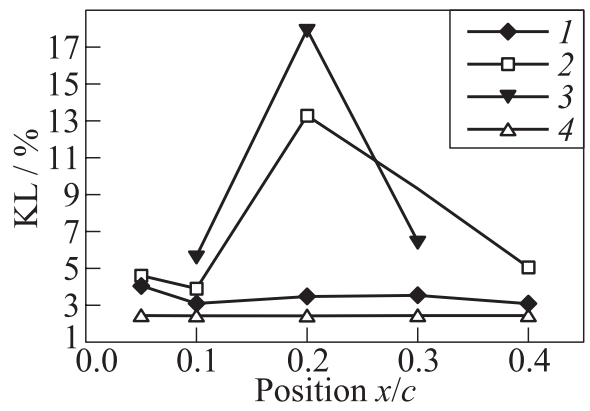

Figure 5 Pressure loss coefficient for different weld positions as computed by the $k-\varepsilon$ realizable (1) and $k-\omega$ SST (2) models in comparison to experiments (3) and clean case (4)

with the earlier results in [3-10]. Some differences between predictions by two turbulence models were found but these discrepancies were smaller than the experimental data scattering for different vanes in the cascade and thus were within the margines of experimental errors. Therefore, it was concluded that both turbulence models predicted the baseline case successfully.

The cases with implemented nonconformances were considerably more challenging for the computations. For certain 2D weld positions, the flow separation was observed in the experiments. This was not surprising since the welding trace size was specially chosen to obtain such effects. A massive stall was observed when the welding trace was located at the position $x / c=0.2$ which was a position of the minimum pressure, i. e., the suction peak on the vane. This is a well known fact that the laminar boundary receptivity to disturbances on airfoils is highest near the pressure minimum (see, e. g., [11]). In the current case, however, the boundary layers were turbulent. The flow separation was also observed for adjacent positions $x / c=0.1$ and 0.3 . Since the prediction of the separation margins is essentially important in the aerodesign, the focus was on these particular weld locations.

Figure 5 shows the pressure loss coefficient obtained by the two turbulence models in comparison with experimental data and the baseline case. Compared to the reference case, the losses in the experiments increase more than a factor of 7 for the central position and about a factor of 5 for the two adjacent positions. However, one can clearly see from this figure that the realizable $k-\varepsilon$ turbulence model does not predict this variation of the loss coefficient. The value of the loss is greatly underpredicted for the positions around the suction peak where the experimental data demonstrate a significant loss increase due to the triggered flow stall. Concerning the results from the $k-\omega$ SST model, the figure shows that this model is capable of predicting the trend and demonstrates a significant 


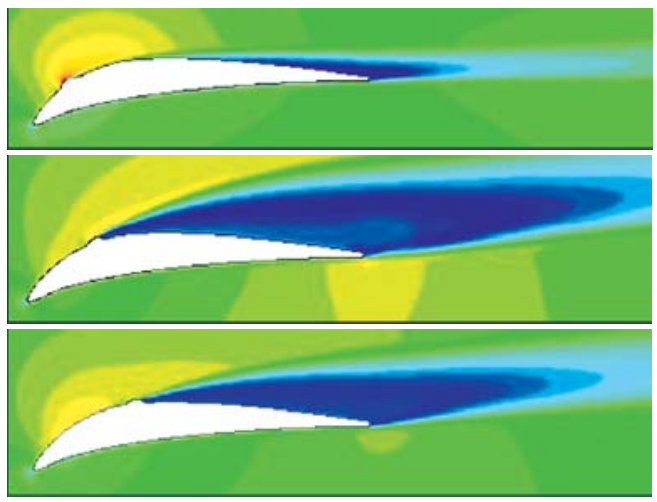

Figure 6 Contours of velocity magnitude near the vane for different weld trace positions: $0.1,0.2$, and 0.3 (from top to bottom). Results of $2 \mathrm{D}$ simulations by the $k-\omega$ SST model. (Refer Chernoray et al., p. 452.)

loss increase at $x / c=0.2$, which is in agreement with the experiments. One can also observe that the results from this model are underpredicting the losses for $x / c=0.1$ and overpredicting for $x / c=0.3$. For the two extreme positions $(x / c=0.05$ and 0.4$)$, there is no experimental data available to compare with. The CFD data reveal a peak at $x / c=0.05$, where the loss increases noticeably with respect to the position $x / c=0.1$ and both turbulence models are predicting a similar result. One possible explanation is that in this zone, the pressure gradient is favourable and both turbulence models seem to perform reasonably well. Concerning the last downstream position, $x / c=0.4$, the pressure losses decrease as compared to the previous position, and both models reflect this reduction. However, the realizable $k-\varepsilon$ model results seem too optimistic and unsafe. It can be noted that for the most downstream positions, the loss decrease is expected since the boundary layer thickness increases and the flow receptivity decreases with downstream distance.

A summary of the results of this section is that the realizable $k-\varepsilon$ model misses the separation completely and gives unsafe results for the weld-type nonconformances. The $k-\omega$ SST model predictions are on the safe margin while instead they can lead to somewhat conservative designs in the unfavourable pressure gradient area.

Details of the flow development are presented in Fig. 6. The flow patterns for different weld positions are shown as predicted by the computations using $k-\omega$ SST model. This figure depicts the contours of the velocity magnitude around the vane. Note that the diagrams show only a part of the computational domain. 
Further comparison between the calculations and experiments is performed in Figs. 7-9. These figures depict the total pressure loss in the wakes for different positions from the vane trailing edge: $0.1,0.25,0.5$, and 0.8 chord. Figure 7 shows the results for the weld location $x / c=0.1$ and Figs. 8 and 9 for the locations $x / c=0.2$ and 0.3 , correspondingly. One can observe that the pressure losses in the OGV cascade are caused primarily by the losses on the vane suction side. Furthermore, one can see that the realizable $k-\varepsilon$ model shows too small wake size in all cases and does not predict the loss increase on the suction side due to the nonconformances. Nevertheless, this model demonstrates reasonably good prediction for the pressure side, as particularly visible in Fig. 9. In general, the predictions by the $k-\varepsilon$ model are poor and the results show almost the same total pressure profiles as for the baseline case without the welds. The results from the $k-\omega$ SST calculations are significantly closer to the experiments. In agreement with the previous discussions, the $k-\omega$ SST model underpredicts the size of the separation for the position $x / c=0.1$ and overpredicts it for $x / c=0.2$ and 0.3. Noticeable is that for the first downstream profile in Fig. 8, the wake size predicted by the $k-\omega$ SST model agrees markedly well with the experiments, while the discrepancies occur further downstream. Thus, the $k-\omega$ SST calcula-

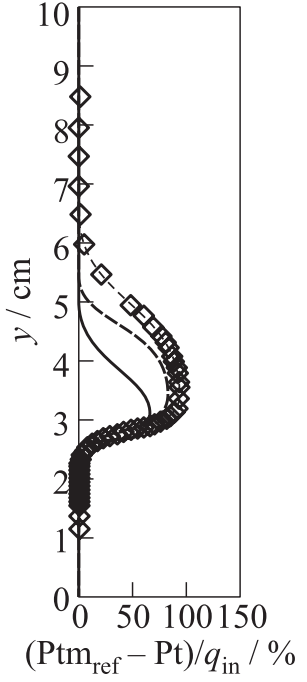

(a)

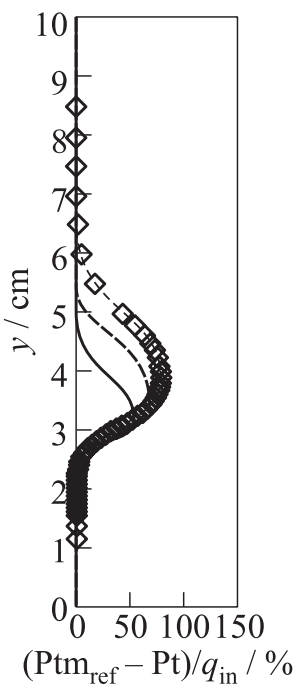

(b)

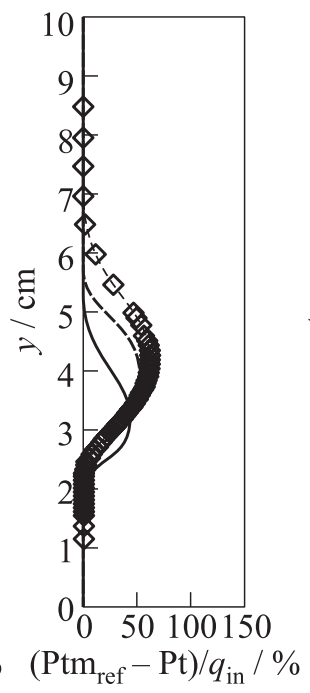

(c)

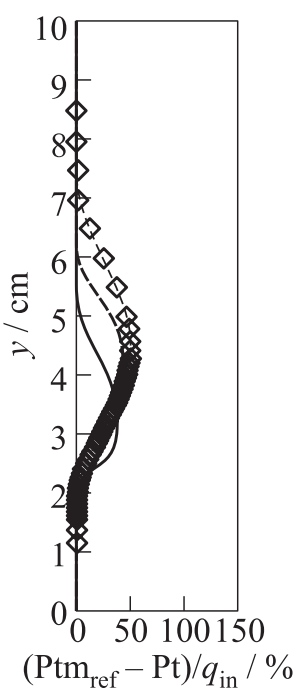

(d)

Figure 7 Weld position $x / c=0.1$. Total pressure loss in wakes in comparison to experimental data shown for different positions from the vane trailing edge: $0.1(a)$, $0.25(b), 0.5(c)$, and $0.8(d)$ chord downstream. Symbols depict experiments and lines calculations by $k-\varepsilon$ realizable model (solid curves) and $k-\omega$ SST model (dashed curves) 


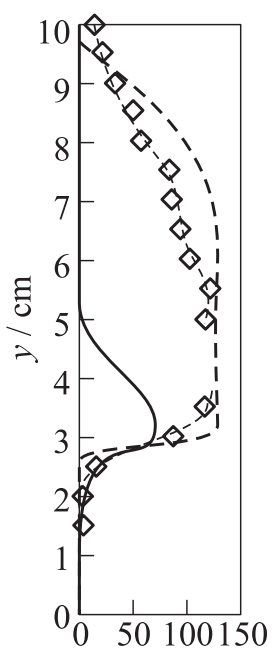

$\left(\mathrm{Ptm}_{\text {ref }}-\mathrm{Pt}\right) / q_{\text {in }} / \%$

(a)

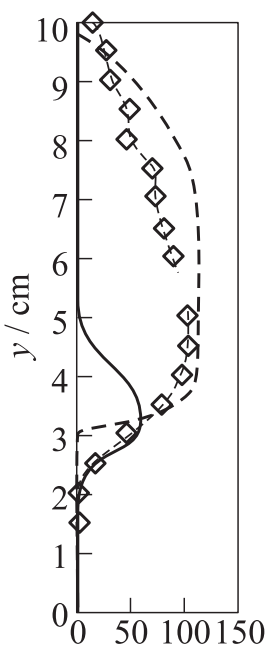

$\left(\mathrm{Ptm} \mathrm{ref}_{\text {ef }}-\mathrm{Pt}\right) / q_{\text {in }} / \%$

(b)

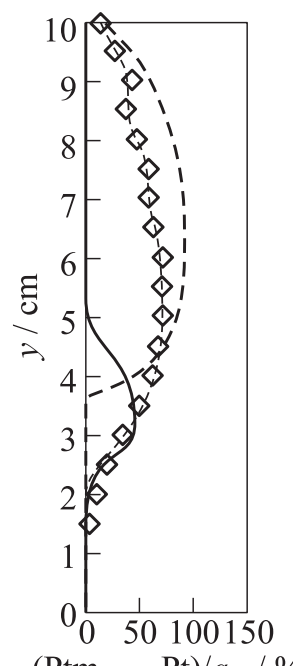

$\left(\mathrm{Ptm}_{\mathrm{ref}}-\mathrm{Pt}\right) / q_{\text {in }} / \%$

(c)

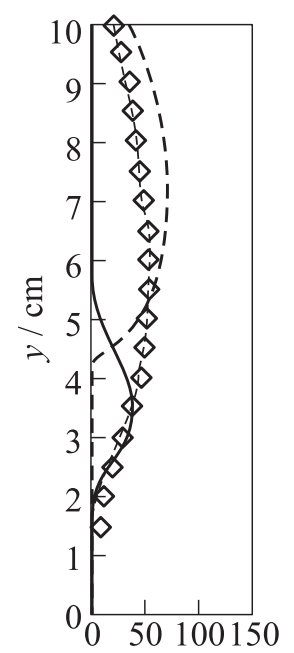

$\left(\mathrm{Ptm}_{\mathrm{ref}}-\mathrm{Pt}\right) / q_{\text {in }} / \%$

(d)

Figure 8 Weld position $x / c=0.2$. See caption of Fig. 7 for details

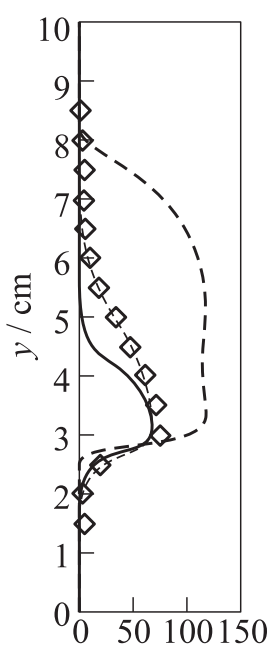

$(\mathrm{Ptm}$ ref $-\mathrm{Pt}) / q_{\text {in }} / \%$

(a)

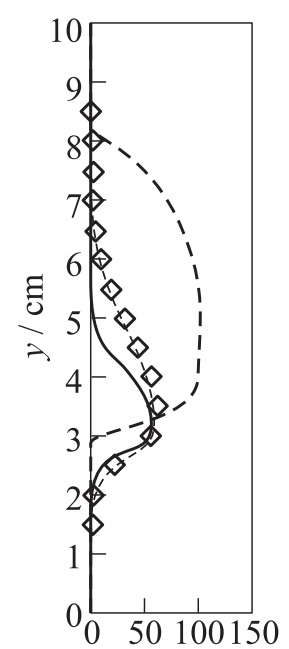

$(\mathrm{Ptm}$ ref $-\mathrm{Pt}) / q_{\text {in }} / \%$

(b)

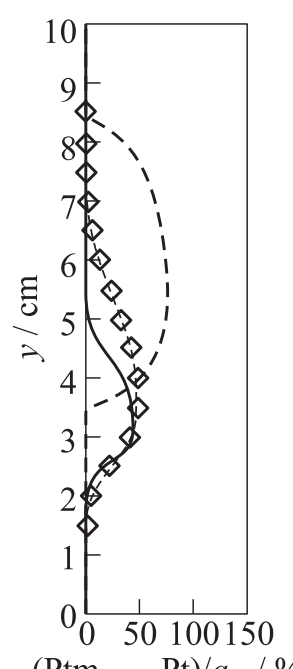

$\left(\mathrm{Ptm}_{\mathrm{ref}}-\mathrm{Pt}\right) / q_{\text {in }} / \%(\mathrm{Ptm}$ ref $-\mathrm{Pt}) / q_{\text {in }} / \%$

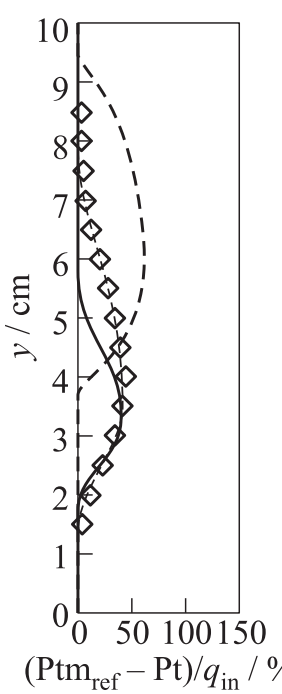

(d)

Figure 9 Weld position $x / c=0.3$. See caption of Fig. 7 for details 
tions demonstrate insufficient wake diffusivity. This fact is remarkable and in contradiction with the previous studies [3] where the turbulence models demonstrated too much diffusivity in the wakes. The problem in the current case is associated with incorrect flow recovery from the separation which seems to be a typical problem of the $k-\omega$ SST model [12]. One can also notice that the wakes in $k-\omega$ SST calculations move rapidly upwards which indicates the flow underturning.

The second part of this work is devoted to the study of nonconformances in the form of $3 \mathrm{D}$ bulges. As was previously explained, the bumps used had two different sizes, a smaller bump with 3-millimeter height and a larger one with 5-millimeter height. Figures 10 and 11 show the results of the calculations by the $k-\omega$ SST model for both cases. In Fig. 10, the isocontours on the vane surface depict streamwise wall-shear stress whereas the isosurfaces show the velocity magnitude from the minimum negative value to $0 \mathrm{~m} / \mathrm{s}$. In this way, the separation zones can be clearly identified. Figure 11 shows the flow pathlines released from the vane surface which visualize the vortical motion in the flow. In the case with 3-millimeter bumps, one can clearly observe a corner vortex and a separation zone near the sidewall. Exactly the same flow structure can be also observed in the baseline case without a bump (not reported here, see, e. g., $[3,9,10])$. In addition, there is a small separation zone just behind the bump and a small-amplitude vortex created by the bump. The change in losses is not detectable by the CFD in this case and of order of the mesh dependency variation. Experiments show a very small loss increase, and in comparison to the initial loss without the bump, the loss changes by a factor of 1.05. In the case with the 5 -millimeter bump, the flow structure predicted by CFD changes

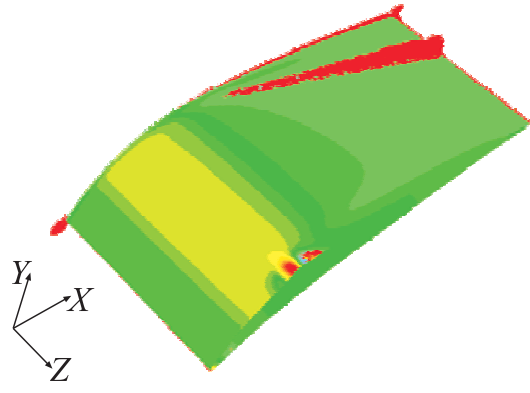

(a)

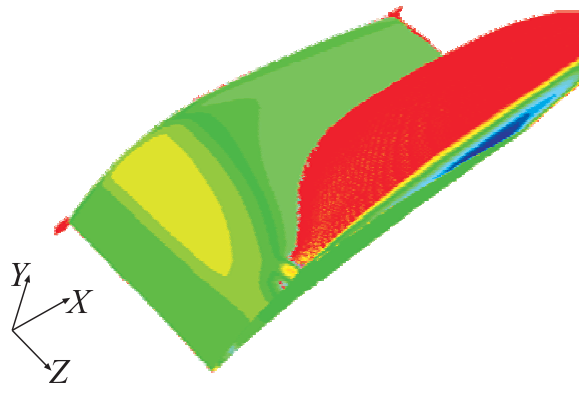

(b)

Figure 10 Predictions by the $k-\omega$ SST model for 3-millimeter bump $(a)$ and 5 -millimeter bump (b). Isocontours on the surface depict $x$ wall-shear stress and isosurfaces show the velocity magnitude from minimum negative value to $0 \mathrm{~m} / \mathrm{s}$ (red). (Refer Chernoray et al., p. 455.) 


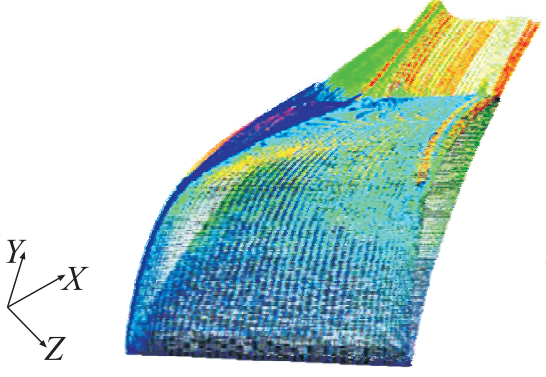

(a)

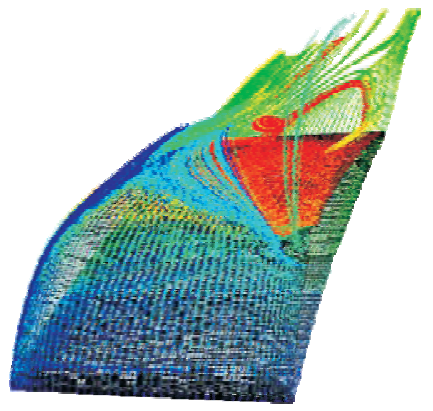

(b)

Figure 11 Flow pathlines released from the vane surface for the 3-millimeter bump $(a)$ and 5-millimeter bump (b). Calculations by the $k-\omega$ SST model. (Refer Chernoray et al., p. 456.)

significantly. One can see that the localized bump caused an extensive flow separation. Due to the induced pressure change by the separation bubble, the corner vortex separation disappeared and the corner vortex size was also reduced. The CFD results in this case predict the loss increase by a factor of 2 compared to the baseline case. In the experiments, the loss increases only by a factor of 1.15 . In general, this case was found essentially challenging for CFD and the solution results changed significantly with grid changes and with changes of the solution history.

The above results clearly demonstrate a possibility for a relatively small spatially localized 3D nonconformance at certain conditions to produce rather significant global flow changes. This happens when the flow separation is triggered. As a result, a dramatic increase in pressure losses occurs in this case. In the experiments, no such global separation from the 3D bumps was observed; however, one can presume that this effect will be observable for bigger bumps, and this will happen since the calculations by the $k-\omega$ SST model have a tendency to overpredict the separation margins.

\section{CONCLUDING REMARKS}

Experiments and CFD calculations of a highly loaded LPT/OGV cascade equipped with simulated surface geometry variations were performed. Two major types of OGV surface nonconformances were simulated and investigated: a $2 \mathrm{D}$ welding trace and a 3D bump (bulge). Computations by two different turbulence models, $k-\omega$ SST and $k-\varepsilon$ realizable, were carried out and validated. Main parameters for the numerical simulations and the boundary conditions at the in- 
let, such as velocity, turbulence length scale, turbulence intensity, and side-wall boundary layer thickness, were matched with experimental conditions in the rig. The results for load distributions, losses, outlet flow angles, and the evolution of the secondary flow-field were monitored and analyzed.

The CFD simulations for the reference clean case revealed a good flow field prediction with both $k-\varepsilon$ realizable and $k-\omega$ SST turbulence models. However, the results appeared to differ essentially for the cases with surface distortions, inducing boundary layer separation.

The $k-\omega$ SST turbulence model was found to predict the locations sensitive to separation and critical bump sizes relatively well. In certain cases, this model behaved far too conservative and overpredicted the flow separation; however, this means that the safe designs will be created based on this model predictions. The $k-\omega$ SST model also demonstrated slightly incorrect flow recovery from the separation which seems to be a typical problem of this model. The realizable $k-\varepsilon$ model predictions were far too optimistic and the flow separation was not predicted. This behavior was particularly observed when the nonconformances were located in the area of unfavourable pressure gradient. For the case with the 3D-bump type nonconformances, high solution sensitivity on the mesh and other calculation parameters was found. This fact is probably due to the sharp separation margin for this geometry.

The results of the present study also show in which way different geometry variations and their positions affect the OGV performance. It is noticeable that even relatively large surface defects can play insignificant role for the OGV performance if they are located at specific locations on the vane surface. However, there is also a range of locations on the OGV surface in the region of the suction side peak where the surface deteriorations influence the aerodynamic properties considerably. This is true both for the simulated 2D welding traces and for the $3 \mathrm{D}$ bumps. A dramatic increase in loss occurs if the flow separation is triggered and if the size of the nonconformance is above a certain critical size. For nonconformances below the critical size, the separation does not occur and the loss change is negligible even for the sensitive locations on the vane around the suction peak. This means that the positions on the OGV surface and corresponding surface treatment can be identified providing a basis for the design and repair rules for the OGVs.

\section{REFERENCES}

1. Hjärne, J., J. Larsson, and L. Löfdahl. 2003. Design of a modern test-facility for LPT/OGV flows. ASME Paper No. GT2003-38083.

2. Hjärne, J., V. Chernoray, J. Larsson, and L. Löfdahl. 2005. Experimental evaluation of the flow field in a state of the art linear cascade with boundary-layer suction. ASME Raper No. GT2005-68399. 
3. Hjärne, J. 2007. Turbine outlet guide vane flows. Ph.D. Thesis. Chalmers University of Technology.

4. Chernoray, V., and J. Hjärne. 2008. Improving the accuracy of multihole probe measurements in velocity gradients. ASME Paper. No. GT2008-50492.

5. Azad, R.S., and S.Z Kassab. 1989. New method of obtaining dissipation. Exp. Fluids 7:81-77.

6. Elsner, J. W., P. Domagava, and W. Elsner. 1993. Effect of finite spatial resolution of hot-wire anemometry on measurements of turbulence energy dissipation. Meas. Sci. Technol. 4:517-23.

7. Kresta, S. M., and P.E. Wood. 1993. The flow field produced by a pitched blade turbine: Characterization of the turbulence and estimation of the dissipation rate. Chem. Eng. Sci. 48(10):1761-74.

8. Merci, B., E. Dick, J. Vierendeels, and C. De Langhe. 2002. Determination of $\varepsilon$ at inlet boundaries. Int. J. Numer. Methods Heat Fluid Flow 12(1):65-80.

9. Ramos Alonso, R. 2009. Non conformance effects on outlet guide vanes. Master Thesis. Chalmers University of Technology.

10. Hjärne, J., V. Chernoray, J. Larsson, and L. Löfdahl. 2007. Numerical validations of secondary flows and loss development downstream of a highly loaded low pressure turbine outlet guide vane cascade. ASME Paper. No. GT2007-27712.

11. Boiko, A.V., G.R. Grek, A. V. Dovgal, and V.V. Kozlov. 2002. The origin of turbulence in near wall flows. Springer.

12. Menter, F. R., M. Kuntz, and R. Langtry. 2003. Ten years of industrial experience with the SST turbulence model. In: Turbulence, heat and mass transfer. Eds. K. Hanjalic, Y. Nagano, and M. Tummers. Begell House Inc. 4:625-32. 